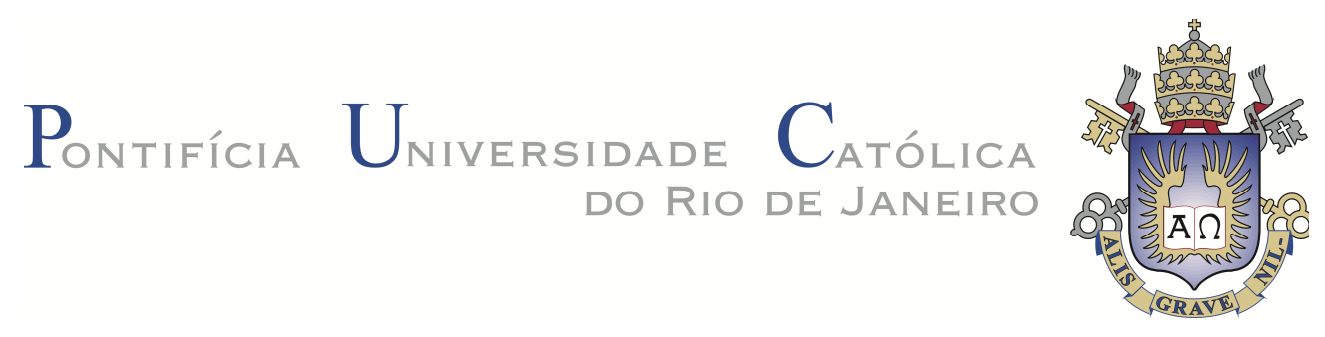

Ruby Lorena Hernández Pico

Influência da microestrutura nas propriedades mecânicas de rochas carbonáticas usando imagens 3D de microtomografia de raios- $X$

Dissertação de Mestrado

Dissertação apresentada como requisito parcial para obtenção do grau de Mestre pelo Programa de PósGraduação em Engenharia Civil do Departamento de Engenharia Civil da PUC-Rio.

Orientador: Prof. Sergio Augusto Barreto da Fontoura

Rio de Janeiro Setembro de 2013 


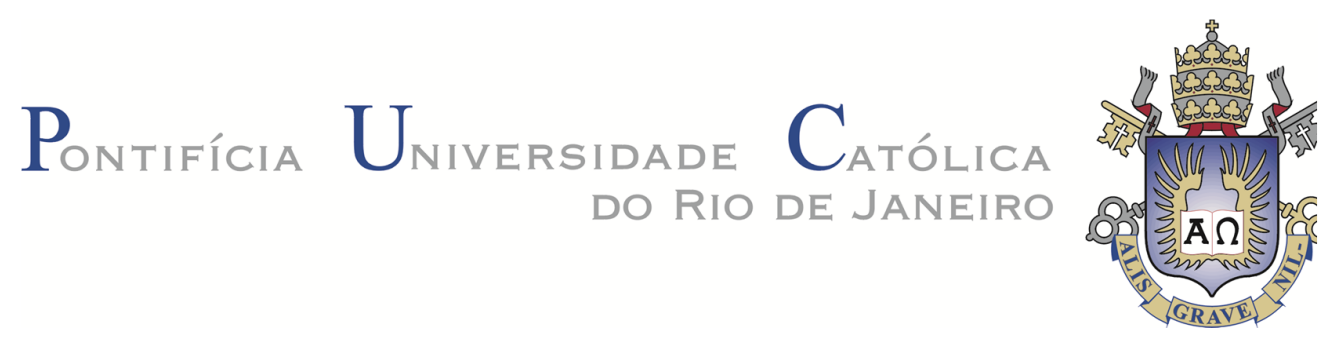

Ruby Lorena Hernández Pico

\section{Influência da microestrutura nas propriedades mecânicas de rochas carbonáticas usando imagens 3D de microtomografia de raios- $X$}

Dissertação apresentada como requisito parcial para obtenção do grau de Mestre pelo Programa de PósGraduação em Engenharia Civil do Departamento de Engenharia Civil do Centro Técnico Científico da PUCRio. Aprovada pela Comissão Examinadora abaixo assinada.

Prof. Sergio Augusto Barreto da Fontoura Orientador Departamento de Engenharia Civil - PUC-Rio

Prof. Sidnei Paciornik Departamento de Ciência dos Materiais Metalúrgica - PUC-Rio

Prof. Celso Romanel Departamento de Engenharia Civil - PUC-Rio

Dr. Erick Slis Raggio Santos CENPES/PETROBRAS

José Eugenio Leal Coordenador setorial do Centro Técnico Científico - PUC-Rio 
Todos os direitos reservados. É proibida a reprodução total ou parcial do trabalho sem autorização da universidade, da autora e do orientador.

\section{Ruby Lorena Hernández Pico}

Graduou-se em Engenharia de Petróleos na UIS (Universidad Industrial de Santander-Colombia) em 2009. Durante a graduação, atuou como pesquisadora na área de geomecânica enfocada a predição de produção de areia em poços produtores de hidrocarbonetos no convenio UIS - ICP (Instituto Colombiano del Petróleo). Atualmente, pesquisadora do Grupo de Tecnologia e Engenharia de Petróleos GTEP-PUC-Rio, nas áreas de análise de estabilidade e caracterização de carbonatos.

Ficha Catalográfica

Hernández Pico, Ruby Lorena

Influência da microestrutura nas propriedades mecânicas de rochas carbonáticas usando imagens digitais 3D de microtomografia de raios- $X$ / Ruby Lorena Hernández Pico; orientador: Sergio Augusto Barreto da Fontoura - 2013.

167 f. il. (color.); $30 \mathrm{~cm}$

Dissertação (mestrado)-Pontifícia Universidade Católica do Rio de Janeiro, Departamento de Engenharia Civil, 2013.

Incluí referências bibliográficas.

1. Engenharia civil - Teses. 2. Microestrutura. 3. Imagens digitais. 4. Microtomografia. 5. Análise numérica. 6. Propriedades mecânicas. 7. Módulo de Young. I. Fontoura, Sergio Augusto Barreto da. II. Pontifícia Universidade Católica do Rio de Janeiro. Departamento de Engenharia III. Título. 
A meus incondicionais companheiros de luta Mãe Maritza Pico A., pai Nazario Hernández F., Irmãos: Laura M. H. P., Danny F. H. P e namorado Elkin O. Mantilla. J. 


\section{Agradecimentos}

Em primeiro lugar a Deus, por seu amor, permissão, bondade e misericórdia; por acompanhar-me nas dificuldades e brindar-me a sabedoria para aprender com elas; por ter colocado e mantido em meu caminho pessoas realmente valiosas; por ensinar-me o verdadeiro valor das coisas simples, o verdadeiro significado da distancia e por brindar-me a força necessária para realizar meus sonhos.

Agradeço à minha mãe, Maritza Pico Ariza, meu grande exemplo de luta e perseverança, por seus inúmeros esforços e sacrifícios para que eu me tornasse a profissional que sou, por manter minha motivação nos momentos certos, por brindar-me seu amor, apoio, orações e companhia incondicional mesmo distante fisicamente. Por sempre ter um espaço para mim apesar das próprias dificuldades, você caminha comigo em todos os momentos. Obrigada por seu nobre ensinamento, você é minha mentora, minha guerreira e heroína. Admiro você, mãe.

Agradeço a meu pai, Nazario Hernández, por dar-me a fortaleza moral, por forjar meu caráter com mão firme na minha infância e adolescência, por ensinar-me a nunca desistir de meus sonhos, por mostrar-me o eterno amor que você sente por seus filhos, por sempre ter uma benção para mim, por dar-me exemplo da imensa 
fé e ser meu guia espiritual, você é uma prova viva da existência dos milagres de Deus.

Gostaria de agradecer a meus irmãos por serem uma de minhas grandes motivações, por lembrar-me que nunca podemos desistir e que as dificuldades fazem os sonhos valer a pena. A minha irmã, Laura Hernández, obrigada por suas orações, amizade e companhia na distancia. A meu irmão por brindar-me sempre o carinho de um coração nobre e seus conselhos.

Gostaria de agradecer a meu querido namorado, Elkin O. Mantilla, você tem me ensinado coisas de valor incalculáveis, você me ensino o verdadeiro sentido do querer, de não desistir, obrigada por não desistir de mim, por acreditar no relacionamento a distancia. Obrigada por permitir-me conhecer um sonhador incansável, de criatividade admirável, um menino sobrevivente no homem imprevisível que você é. Sempre estarei grata pelo apoio e amor incondicional. Você é muito mais do que imagina para mim e das palavras escritas aqui.

Agradeço a minha família em geral por serem motivação para ser cada vez melhor. Agradeço especialmente a minha tia querida, Maria C. Hernández e a meu tio, Clodomiro Torres por serem apoios em etapas decisivas de minha vida.

Agradeço especialmente ao professor Sergio A. B. Fontoura pela valiosa oportunidade e pela confiança depositada em minhas capacidades e em minha pessoa desde nossa primeira conversa. Agradeço sua amizade, paciência, apoio e incentivo tanto acadêmico como pessoal. Obrigada por acolher-me e disponibilizar as ferramentas e infraestrutura necessária do GTEP para o desenvolvimento deste trabalho.

Gostaria de agradecer a Guilherme Lima Righetto por sua amizade, paciência, tempo, disponibilidade e entusiasmo, por compartilhar seu valioso conhecimento e suas brilhantes ideais durante esta dissertação. Sem você não teria sido possível.

Agradeço a Débora Pilotto por sua amizade, por compartilhar e transmitir-me seu conhecimento e aprendizado. 
Agradeço a cada uma das personas do grupo de tecnologia e engenharia de petróleo (GTEP-PUC-Rio) por serem parte importante nesta ultima etapa de meu mestrado, por abrir as portas do grupo e acolher-me da melhor forma possível, sempre estarei grata com cada um de vocês.

Agradeço a Talita Miranda e a Vivian Marchesi pela amizade, seus conselhos foram parte vital para mim nesta etapa.

Agradeço a toda equipe do Laboratório de Processamento Digital de Imagens (LPDI). Especialmente ao professor Sidnei Paciornik por brindar-me seu conhecimento, o laboratório e por permiti-me assistir a suas excelentes aulas.

Agradeço a Rosimeria Silva e Álvaro Talavera pela ajuda e sua sincera amizade.

Gostaria de agradecer a Darwin Mateus e a Juan David por sua amizade e apoio mútuo. Sem dúvidada uma excelente equipe Colombiana nesta etapa.

Agradeço a minha amiga, Karina Silva Salas, pelo acompanhamento e apoio mútuo desde a graduação.

Agradeço a todos meus amigos que, mesmo desde à distancia torcem por meu sucesso, especialmente a Alexander Martinez, por seu apoio, carinho e orientação incondicional.

Agradeço ao Departamento de Engenharia civil, a cada um dos professores que foram parte de minha formação.

Agradeço a Grupo de Tecnologia e Engenharia de Petróleo (GTEP-PUC-Rio) pela concessão do complemento de bolsa de mestrado.

Agradeço à PUC-Rio.

Agradeço à Coordenação de Aperfeiçoamento de Pessoal de Nível SuperiorCAPES pela concessão da bolsa de mestrado. 


\section{Resumo}

Hernández Pico, Ruby Lorena. Fontoura, Sergio Augusto Barreto. Influência da microestrutura nas propriedades mecânicas de rochas carbonáticas usando imagens 3D de microtomografia de raios-X. Rio de Janeiro, 2013. 167p. Dissertação de Mestrado - Departamento de Engenharia Civil, Pontifícia Universidade Católica do Rio de Janeiro.

Reservatórios carbonáticos contêm entre 50 e $60 \%$ de petróleo e gás do mundo. No entanto, estas rochas têm apresentado problemas operacionais consideráveis durante as etapas de perfuração e produção. Tais problemas são originados pelas dificuldades na caracterização adequada destas rochas em função da complexa distribuição espacial de sua micro e macro estrutura. Enquanto ambas as escalas possuem importância no entendimento do comportamento de carbonatos, o presente trabalho trata da análise da microestrutura. Nesse âmbito, a proposta deste trabalho integra o processamento e análises de imagens digitais adquiridas mediante microtomografia de raios-X, elaboração de malhas de elementos finitos e simulação numérica de forma a prever propriedades elásticas, com o objetivo de correlacionar a microestrutura e o módulo de Young. Foram utilizadas imagens digitais de amostras de afloramento (travertinos) considerados como possíveis análogas a rochas carbonáticas de reservatório. A metodologia implementada permitiu obter sub-amostras que incluem uma gama de microestruturas e porosidades numa única amostra para assim simular numericamente o módulo de Young. Os resultados dos ensaios virtuais foram comparados com os resultados de ensaios reais executados em amostras da mesma rocha e dados encontrados na literatura. A aplicação da metodologia e os resultados obtidos indicam o potencial e as limitações atuais desta técnica. Conclui-se neste estudo que o valor do módulo de Young das amostras simuladas numericamente é afetado pela distribuição espacial, conectividade dos poros e pela microporosidade da rocha. Os valores encontrados no ensaio virtual tendem a serem superiores aos obtidos em ensaios reais devido à dificuldade de inserir, no modelo de micro estrutura, os contatos entre os grãos assim como regiões mais compressíveis não determinadas na aquisição das imagens.

\section{Palavras-chave}

Microestrutura; imagens digitais; microtomografia; análise numérica; propriedades mecânicas; módulo de Young. 


\section{Abstract}

Hernandez Pico, Ruby Lorena. Fontoura, Sergio Augusto Barreto (Advisor). Influence of microstructure on mechanical properties of carbonate rocks using 3D digital images of X-ray microtomography. Rio de Janeiro, 2013. 167p. M.SC. Dissertation - Departamento de Engenharia Civil, Pontifícia Universidade Católica do Rio de Janeiro.

Carbonate reservoirs contain between 50 and $60 \%$ of oil and gas in the world. However, these rocks have presented considerable operational problems during drilling and production steps. Such problems are caused by the difficulties in proper characterization of these rocks due the complex spatial distribution of their micro and macrostructure. Although both scales have importance in understanding the behavior of carbonates, the present work deals with the analysis of the microstructure. In this context, the proposal of this work integrates the processing and analysis of digital images acquired by x-ray microtomography, finite element mesh generation and numerical simulations to predict elastic properties in order to correlate the microstructure and the Young's modulus. We used digital images of samples of outcrop (travertine) considered as a possible analogous to carbonate rocks of reservoir. The methodology implemented allowed get sub-samples that include a range of microstructures and porosities in a single sample to determine numerically the Young's modulus. The virtual test results were compared with laboratory test results performed on samples from the same rock and data found in the literature. The application of the methodology and the results obtained indicate the potential and current limitations of this technique. This study concluded that the value of Young's modulus of numerically simulated samples is affected by spatial distribution, pore connectivity and microporosity of these rocks. The values found in the virtual test tend to be higher than those obtained in laboratory tests due to the difficulty of inserting, in the model of micro structure, contacts between the grains as well as more compressible regions not certain on the acquisition of the images.

\section{Keywords}

Microstructure; digital imaging; microtomography; numerical analysis; mechanical properties; Young's modulus. 


\section{Sumário}

1 Introdução 19

1.1. Motivação e objetivo 19

1.2. Estrutura da dissertação 20

2 Revisão Bibliográfica $\quad 21$

2.1. Importância das rochas carbonáticas 21

2.2. Definição de rochas carbonáticas 21

2.2.1. Rochas carbonáticas detríticas 22

2.2.2. Rochas carbonáticas orgânicas $\quad 24$

2.3. Processos de formação: Sedimentação e diagênese 25

2.4. Reservatórios carbonáticos 28

2.4.1. Formação de reservatórios carbonáticos 30

2.5. Classificação das rochas carbonáticas 33

2.6. Principais características das rochas carbonáticas 34

2.6.1. Características geológicas e petrofísicas 35

2.6.2. Características mecânicas 39

2.6.3. Microestrutura, propriedades elásticas e mecânicas. 44

2.7. Técnicas de estudo das rocas carbonáticas 49

$\begin{array}{ll}\text { 2.7.1. Microtomografia } & 50\end{array}$

2.7.2. Processamento de imagens e análise digital de imagens (PADI) 52

2.7.3. Geração de malhas a partir de volumes tridimensionais 58

2.7.4. Análise de elementos finitos 63

2.7.5. Rochas carbonáticas sob o ponto de vista de simulação 68

3 Materiais e métodos $\quad 74$

3.1. Métodos $\quad 74$

3.2. Definição de amostras $\quad 75$

3.2.1. Estudos preliminares $\quad 75$

3.3. Aquisição de imagens 86

3.4. Processamento e análises 88

3.5. Geração de malhas $\quad 95$

3.6. Aplicação de análise de Elementos Finitos 98 
4 Resultados

104

4.1. Aquisição das amostras 104

4.2. Processamento e análises de imagens 106

4.2.1. Sub - amostragem em imagens digitais 106

4.2.2. Pré-processamento, processamento, análise e visualização no CTAN 108

4.2.3. Pré-processamento, processamento, análises e visualização no ScanIP.111

4.3. Geração das malhas no volume 3D 120

4.4. Modelo computacional e método de elementos finitos 127

4.5. Análise e avaliação de resultados 135

5 Considerações finais $\quad 150$

5.1. Conclusões 150

5.2. Recomendações 153

$\begin{array}{ll}\text { Referências Bibliográficas } & 154\end{array}$

Apêndice I Sub-amostragem digital e modelos tridimensionais rocha-poro. 163 


\section{Lista de figuras}

Figura 2-1. Principais grãos aloquímicos das rochas carbonáticas. .................. 23

Figura 2-2. Rochas carbonáticas orgânicas. ................................................... 24

Figura 2-3. Diagrama de pressão-temperatura e regimes de diagênese............ 26

Figura 2-4. Origem dos reservatórios carbonáticos........................................ 32

Figura 2-5. Fase inicial da formação do Atlântico Sul......................................... 33

Figura 2-6. Distribuição de rochas carbonáticas. ........................................... 33

Figura 2-7. Relação: tamanho do grão, seleção e porosidade em areias

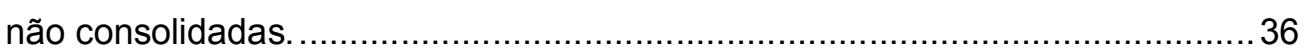

Figura 2-8. Estrutura de deposição em rochas detríticas..................................38

Figura 2-9. Influência das propriedades fundamentais sobre a porosidade........ 38

Figura 2-10. Módulo de Young frente à porosidade.. ..................................... 40

Figura 2-11. Propriedades mecânicas e ultra-sons com porosidade ................ 43

Figura 2-12. Resistência para travertinos bandeados, direção de bandas paralelas e perpendiculares á direção da carga............................................. 44

Figura 2-13. Curvas tensão-deformação registrado para travertinos (ALHTR, CLASTR e ALBTR) e tufo (GODTUF). .......................................... 44

Figura 2-14. Microestrutura, dependência e influência do material. ................... 45

Figura 2-15. Fatores que controlam as propriedades físicas das rochas carbonáticas reservatórios.......................................................................... 46

Figura 2-16. Módulo de Young medio frente ao algoritmo de raio medio de

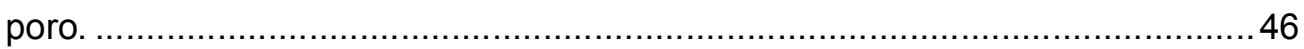

Figura 2-17. Comportamento elástico - plástico de rochas carbonáticas em função de a dissolução e da temperatura.

Figura 2-18. Curvas de UCS em função de $V_{p}$. Curvas $B$ e $E$ definidas para calcários.

Figura 2-19. Curvas de UCS em função de $V_{p}$, diversas litologias $e$ correlações para um mesmo tipo de rocha..

Figura 2-20. Fluxograma do processo de microtomografia de raios-X.

Figura 2-21. Sequência padrão do processamento e análises de imagens........53

Figura 2-22. Procedimentos de pré-processamento no domínio do espaço real. 54

Figura 2-23. Operações pontuais 55

Figura 2-24. Operações Locais. 
Figura 2-25. Pós-processamento: Extração de atributos

Figura 2-26. Etapas gerais de geração de malhas tridimensionais a partir

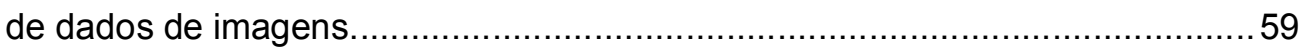

Figura 2-27. Cubos de triangulação..

Figura 2-28. Resultados de propriedades elásticas para carbonatos homogêneos sob três condições de saturação. 71

Figura 2-29. Comparação entre módulos volumétrico e cisalhante

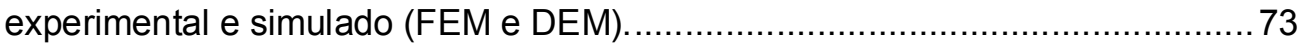

Figura 3-1. Metodologia geral desenvolvida............................................ 74

Figura 3-2. Amostras coletadas de travertino da bacia de Itaboraí.....................76

Figura 3-3. Placas de travertino Romano. ...................................................... 77

Figura 3-4. Placas de travertino Turco................................................... 77

Figura 3-5. Difração de raios-X, Travertino de Itaboraí: a) TRI-01, b) TRI-

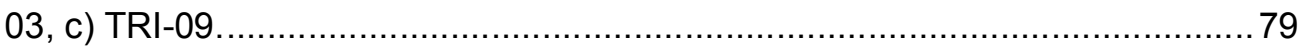

Figura 3-6. Corpos de prova antes e depois do ensaio: (a) TRI-04 e (b)

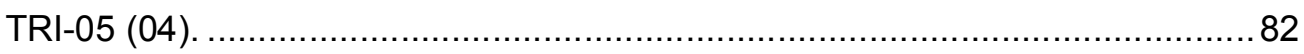

Figura 3-7. Contato entre os grãos da amostra TRI-05................................ 82

Figura 3-8. Difração de raios-X: a) Travertino Romano TRR-01 e b)

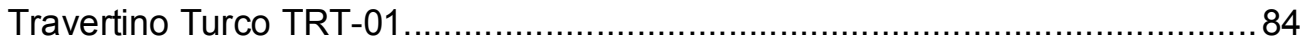

Figura 3-9. Amostras de travertinos: a) TRI-09, b) TRR-01, c) TRT-01 .............. 86

Figura 3-10. Microtomógrafo SkysCan 1173 utilizado no programa experimental: a) vista frontal do equipamento e b) porta amostras. ...................88

Figura 3-11. Metodologia para processamento e análises das imagens digitais dos travertinos microtomografados.

Figura 3-12. Parâmetros específicos utilizados do programa CTAN. Ф:

Porosidade.

Figura 3-13. Parâmetros específicos utilizados do programa ScanIP. Ф:

Porosidade.

Figura 3-14. Amostra de rocha submetida à compressão uniaxial....................98

Figura 3-15. Curva Tensão-deformação... ................................................. 100

Figura 3-16. Fluxograma de etapas de simulação de aplicação de deslocamento.

Figura 4-1. Amostra irregular Travertino Romano. a) Fotografia da amostra.

b) Imagem 3D da amostra microtomografada. 105

Figura 4-2. Amostra irregular Travertino Turco. a) Fotografia da amostra. b) Imagem 3D da amostra microfotografada. 105 
Figura 4-3. Amostra irregular Travertino de Itaboraí. a) Fotografia da amostra. b) Imagem 3D da amostra microfotografada.

Figura 4-4. Sub-amostragem digital: a) Amostra para ensaio de compressão simples. Normas ISRM (2007), b) Análise digital da geometria da amostra e c) Sub-amostragem

Figura 4-5. Fissura presente na amostra de travertino turco microtomografada. Imagem 2D.

Figura 4-6. Sub-amostragem: a) Imagem 3D da amostra irregular do travertino Romano microtomografado. b) Sub-amostra TRR-1. C) Subamostra TRR-2. d) Sub-amostra a TRR-3.

Figura 4-7. Pré-processamento, sub-amostra de travertino turco: a ) Imagem circular 2D antes do filtro, b) Imagem circular 2D depois do filtro , c) segmentação (50 - 255) modelo da rocha, d) segmentação (0-50) modelo dos poros.

Figura 4-8. Comparação dos filtros de ScanIP, TRT, imagem 2D: a) Imagem original, b) Bilateral Filter, c) Mean Filter, d) Median Filter. Figura 4-9. Comparação dos filtros de ScanIP, seção ampliada TRT, imagem 2D: a) Imagem original, b) Bilateral Filter, c) Mean Filter, d) Median Filter.

Figura 4-10. Segmentação da microestrutura no programa ScanIP. TRR-2.

Vista semitransparente: a) segmentação da rocha, b) segmentação dos poros

Figura 4-11. Segmentação da microestrutura no programa ScanIP. TRT-3:

a) Imagem original. b) Imagem binária ressaltando poros e rocha.

Figura 4-12. Modelo da amostra do travertino de Itaboraí no programa ScanIP . a) Fotografia de amostra real. b) Modelo da amostra, em 3D.

Figura 4-13. Volumes 3D de sub-amostra dos três travertinos: a) TRI-1, b)

TRR-1, c) TRT-1.

Figura 4-14. Volumes 3D das sub-amostras: a) TRI-1, b) TRR-1, c) TRT-1 ..... 116

Figura 4-15. Visualização 3D, sub-amostras TRI-1, TRR-1, TRT-1 a)

Visualização externa modelos rocha e poros, b) visualização do sistema poroso interno.

Figura 4-16. Histograma de frequência de diâmetros de poros das subamostras de travertino Romano.

Figura 4-17. Histograma de frequência de diâmetros de poros das subamostras de travertino Turco 
Figura 4-18. Visualização 3D sub-amostra TRT-3. Em vermelho, poros vugurales, em azul, poros tipo intergranular.

Figura 4-19. Vista superior da sub-amostra TRT-3. Malha gerada no algoritmo FEGrid: a) Malha tipo "voxels". b) Malha tipo suavizado e c) Malha gerada com algoritmo FEFree.

Figura 4-20. Visualização 3D do volume total para a sub-amostra TRT-3.

Malha gerada no algoritmo FEGrid: a) Malha tipo "voxels", 8.8e6 elementos, b) Malha tipo suavizado, $1.3 e 7$ elementos e c) Malha gerada no algoritmo FE Free, 9.1e6 elementos.

Figura 4-21. Visualização 3D interna do volume total para a sub-amostra TRT-3. Malha gerada no algoritmo FE Grid: a) Malha tipo "voxels" b) Malha tipo suavizado "smoothed" e c) Malha gerada no algoritmo FEFree 124

Figura 4-22. Curva tensão-deformação: TRI-1 ........................................... 129

Figura 4-23. Curva tensão-deformação: TRT-3, "resample" 80 ...................... 130

Figura 4-24. Curva tensão-deformação: TRI-3, "resample" 60. …................... 130

Figura 4-25. Curva tensão-deformação: TRI-3, "resample" 50....................... 131

Figura 4-26. Curva tensão-deformação: TRR-1 ....................................... 131

Figura 4-27. Curva tensão-deformação: TRR-2 …........................................ 132

Figura 4-28. Curva tensão-deformação: TRR-3 .......................................... 132

Figura 4-29. Curva tensão-deformação: TRT-1 ........................................... 133

Figura 4-30. Curva tensão-deformação: TRT-2 .......................................... 133

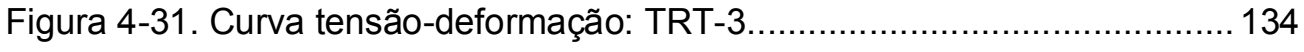

Figura 4-32. Curva tensão-deformação: TRT-4 ......................................... 134

Figura 4-33. Curva tensão-deformação: TRT-5......................................... 135

Figura 4-34. Comparativo: curvas tensão-deformação: TRI-1. Curvas vermelha e lilás obtidas em laboratório, curva azul obtida em simulação

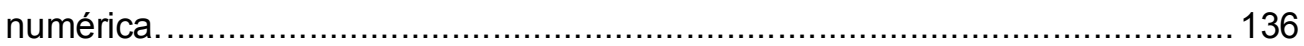

Figura 4-35. Metodologia proposta para trabalhos futuros. ........................... 137

Figura 4-36. Visualização 3D da rocha e o sistema poroso com aplicação de "Resample": a) fator 0, b) fator 80 , c) fator 60 e d) fator 50 . .

Figura 4-37. Diferença na densidade das malhas com aplicação de "Resample": a) fator 0, b) fator 80, c) fator 60 e d) fator 50 .

Figura 4-38. Comparativo: Curvas tensão-deformação para TRT-3 com manipulação de resolução.

Figura 4-39. Comparativo: Curvas Tensão-Deformação para sub-amostras de travertino Romano. 
Figura 4-40. Comparativo: Curvas Tensão-Deformação para sub-amostras de travertino Turco

Figura 4-41. Visualização 3D do TRI-1: a) modelo semitransparente da rocha antes da simulação, b) modelo da rocha após a simulação.

Figura 4-42. Visualização 3D do TRR-1: a) modelo semitransparente da rocha antes da simulação, b) modelo da rocha após a simulação. 142

Figura 4-43. Visualização 3D do TRR-2: a) modelo semitransparente da rocha antes da simulação, b) modelo da rocha após a simulação.

Figura 4-44. Visualização 3D do TRR-3: a) modelo semitransparente da rocha antes da simulação, b) modelo da rocha após a simulação.

Figura 4-45. Visualização 3D do TRT-1: a) modelo semitransparente da rocha antes da simulação, b) modelo da rocha após a simulação.

Figura 4-46. Visualização 3D do TRT-2: a) modelo semitransparente da rocha antes da simulação, b) modelo da rocha após a simulação. 145

Figura 4-47. Visualização 3D do TRT-3: a) modelo semitransparente da rocha antes da simulação, b) modelo da rocha após a simulação.

Figura 4-48. Visualização 3D do TRT-4: a) modelo semitransparente da rocha antes da simulação, b) modelo da rocha após a simulação.

Figura 4-49. Visualização 3D do TRT-5: a) modelo semitransparente da rocha antes da simulação, b) modelo da rocha após a simulação.

Anexo I. 1. Sub-amostragem: a) Imagem 3D da amostra irregular do travertino Romano microtomografado. b) Sub-amostra TRI-1.

Apêndice I. 2. Sub-amostragem: a) Imagem 3D da amostra irregular do travertino Turco microtomografado; b), c), d) e e) Sub-amostras cilíndricas. 164 Apêndice I. 3. Modelos tridimensionais (volume cilíndrico) das subamostras do travertino Turco.

Apêndice I. 4. Visualização 3D, sub-amostras TRR-2 e TRR-3 a) Visualização externa modelos rocha e poros, b) visualização do sistema poroso interno.

Apêndice I. 5. Visualização 3D, sub-amostras TRT-3, TRT-4 e TRT-5 a) Visualização externa modelos rocha e poros, b) visualização do sistema poroso interno. 


\section{Lista de tabelas}

Tabela 2.1. Escalas e técnicas no estudo estrutural.

Tabela 2.2. Comparação de módulos simulados e medidos. Usando o modelo Hetzian.

Tabela 2.3. Comparação de módulos simulados e medidos. Usando o modelo Hookean.

Tabela 3.1. Resultados dos ensaios de compressão simples nos corpos de prova de travertino de Itaboraí.

Tabela 3.2. Resultados dos constituintes presentes nas lâminas

petrográficas de travertinos romanos.

Tabela 3.3. Resultados dos constituintes presentes nas lâminas petrográficas de travertinos turcos.

Tabela 3.4. Faixa de variação de propriedades índice para TRR e TRT.

Tabela 3.5. Resultados da análise da porosidade realizada nos modelos 3D gerados a partir das imagens de microtomografia.

Tabela 3.6. Resultados dos ensaios de esclerometria para travertinos

Romano e Turco.

Tabela 3.7. Parâmetros utilizados no microtomógrafo para as amostras analisadas.

Tabela 3.8. Características de imagens bidimensionais de microtomografia.

Tabela 3.9. Parâmetros de descrição e avaliação da qualidade de elementos tetraédricos.

Tabela 3.10. Módulos, densidades e velocidades de onda da Calcita.

Tabela 3. 11. Módulos Volumétricos, de cisalhamento, Young e coeficiente de Poisson da Calcita.

Tabela 4.1. Dimensões das sub-amostras cilíndricas para os travertinos.

Tabela 4.2. Análises de porosidade no programa CTAN para imagens

digitais das amostras de travertinos.

Tabela 4.3. Análises de porosidade no programa CTAN para imagens digitais das amostras de travertinos.

Tabela 4.4. Análise de porosidade no programa CTAN para sub-amostras cilíndricas.

Tabela 4.5. Análises de porosidade no programa ScanIP para sub-amostras cilíndricas. 
Tabela 4.6. Informação de parâmetros de malhas geradas para os subamostras a partir de algoritmos FEGrid e FEFree.

Tabela 4.7. Informação de parâmetros de malhas geradas para as subamostras a partir do algoritmo FEGrid com manipulação da resolução.

Tabela 4.8. Manipulação da resolução: aplicação de "Resample" para TRR-

1.

Tabela 4.9. Propriedades do principal componente mineralógico: Calcita.

Tabela 4.10. Deslocamentos calculados para as sub-amostras.

Tabela 4.11. Dados obtidos para os modelos do TRT-3 com manipulação da resolução.

Tabela 4.12. Discrepância nos resultados para os modelos do TRT-3.

Tabela 4.13. Porcentagens de porosidade e módulo de Young para subamostras do travertino Romano.

Tabela 4.14. Porcentagens de porosidade e módulo de Young para subamostras do travertino Turco.

Tabela 4.15. Avaliação de resultados: Comparativo dados obtidos por método de elementos finitos com dados de métodos experimentais e literatura.

Tabela 4.16. Tempo requerido para a simulação numérica das sub-amostras dos três tipos de travertinos com manipulação de resolução. 